\title{
Observations of very high energy emission from B2 $1215+30$ with VERITAS
}

\author{
Heike Prokoph for the VERITAS Collaboration ${ }^{1}$ \\ DESY, Platanenallee 6, 15738 Zeuthen, Germany
}

\begin{abstract}
We present results of 45 hours of data taken from January to June 2011 with VERITAS above $200 \mathrm{GeV}$ in the direction of B2 $1215+30$. The blazar was clearly detected with a constant flux of $F(E>200 \mathrm{GeV})=(8.0 \pm 0.9) \times 10^{-12} \mathrm{~cm}^{-2} \mathrm{~s}^{-1}$. The energy spectrum can be fitted using a power-law with index $-3.6 \pm 0.5$. Simultaneous multi-wavelength data are also presented, and are used to model the spectral energy distribution with a one-zone leptonic model.
\end{abstract}

Keywords: AGN, gamma rays, non-thermal, VERITAS

PACS: $98.54 . \mathrm{Cm}$; 95.85.Pw; 98.70.Rz; 98.54.-h

\section{INTRODUCTION}

Blazars (BL Lac objects and flat spectrum radio quasars) are the most extreme class of active galactic nuclei. Their jet is aligned closely with the line-of-sight and they show variability at all wavelengths and time scales. One of the first BL Lac type objects to be identified was B2 1215+30 [1] (also known as 1ES 1215+303 or ON 325). Its redshift is uncertain with two different values found in literature: $\mathrm{z}=0.130$ [2] and $\mathrm{z}=0.237$ [3].

At very high energies (VHE; E $>100 \mathrm{GeV}$ ) B2 1215+30 was first detected by MAGIC in 2011 [4]. VERITAS also observed the source, which is in the same field of view as two other VHE blazars: 1ES 1218+304 and W Comae. The observations of B2 1215+30 with VERITAS in 2011 are reported on here.

\section{VERITAS OBSERVATIONS AND RESULTS}

VERITAS is an array of four imaging atmospheric Cherenkov telescopes located in southern Arizona, sensitive to gamma-ray energies from $100 \mathrm{GeV}$ to about $30 \mathrm{TeV}$, with a field of view of $3.5^{\circ}$. For more details on the VERITAS instrument see [5].

VERITAS observed the sky towards B2 1215+30 for more than 45 hours between January and June 2011, including dedicated pointings and observations of 1ES 1218+304, which is only $0.76^{\circ}$ away. After quality selection and acceptance correction, a total of 38 hours of data remained. The data were analyzed with pre-defined cuts, optimized for a $5 \%$ Crab Nebula-like source. The signal was extracted using a ring background model with an ON region of $\theta^{2}$ radius of $0.008 \mathrm{deg}^{2}$ centered on the position of B2 1215+30.

${ }^{1}$ http://veritas.sao.arizona.edu/ 
In this ON region, 193 excess events have been detected with a significance of $10.4 \sigma$ (using Eq. 17 in [6]). The flux above $200 \mathrm{GeV}$ is found to be $(8.0 \pm 0.9) \times$ $10^{-12} \mathrm{~cm}^{-2} \mathrm{~s}^{-1}$, corresponding to about $3 \%$ of the Crab Nebula flux above the same energy threshold. The derived differential photon spectrum can be fitted by a power law: $\mathrm{dN} / \mathrm{dE}=F_{0}\left(E / E_{0}\right)^{-\alpha}$, with $F_{0}=(2.3 \pm 0.5) \times 10^{-11} \mathrm{~cm}^{-2} \mathrm{~s}^{-1}, E_{0}=300 \mathrm{GeV}$, and $\alpha=3.6 \pm 0.5$. This is compatible with the 2011 MAGIC results reported in [7].

\section{MULTI-WAVELENGTH OBSERVATIONS}

Simultaneous multi-wavelength data have been taken by Fermi-LAT in the high energy regime, Swift-XRT in X-rays, Swift-UVOT in the UV/optical, and with Super-LOTIS and MDM in the optical.

The Fermi-LAT analysis was performed using the standard LAT ScienceTools (version v9r23p1) and P7SOURCE_V6 instrument response functions together with the recommended cuts and background files. A binned maximum-likelihood method was applied to the data in order to extract the light curve (shown in Fig. 1) and the spectrum for the first half of 2011.

Swift-XRT observed B2 1215+30 during January and in March/April 2011. The analysis was performed with HEASoft, XSPEC v12.6.0 and the recommended response files. The spectrum was fitted with an absorbed power law while the Galactic column density was fixed to $N_{H}=1.74 \times 10^{20} \mathrm{~cm}^{-2}$ [9].

In addition to the Swift-UVOT data, MDM took data in May 2011 in the R, I and V band, while Super-LOTIS monitored the variability of B2 $1215+30$ in the R-band. The data reduction of the optical and UV data sets followed standard methods.

In Fig. 1 the light curves for the first half of 2011 are shown. In the VHE regime, no variability could be detected in the monthly binned VERITAS light curve for energies above $200 \mathrm{GeV}\left(\chi^{2} / n d f=4.7 / 5\right)$. Even though variability has been measured by FermiLAT in the past [8], the bi-weekly binned light curve above $200 \mathrm{MeV}$ contemporaneous to the VERITAS observation is compatible with being constant. In X-rays, the blazar is found to be brighter and harder in January than in March/April 2011. Based on those different X-ray states, two SEDs have been extracted which are shown in Fig. 2.

For the January SED the highest X-ray flux (on MJD 55565) was used, together with simultaneous UVOT data. To represent the low X-ray state of the source in Mar/Apr, a combined fit of the XRT data was performed (using all available data from MJD 5567355686). This was accomplished by simultaneous UVOT data and quasi-simultaneous MDM data from May 2011. Since no variability was detected in the high-energy regime in 2011, contemporaneous data from VERITAS and Fermi-LAT are used (MJD 55560 55720). Radio data were retrieved using the NED website [10].

\section{MODELING OF THE SPECTRAL ENERGY DISTRIBUTION}

The extracted quasi-simultaneous SEDs are modeled with a synchrotron self-Compton (SSC) model [11], which takes the intergalactic absorption into account using the model of Finke et al. [12]. Within the model the low-energy emission (from radio to $\mathrm{X}$ - 


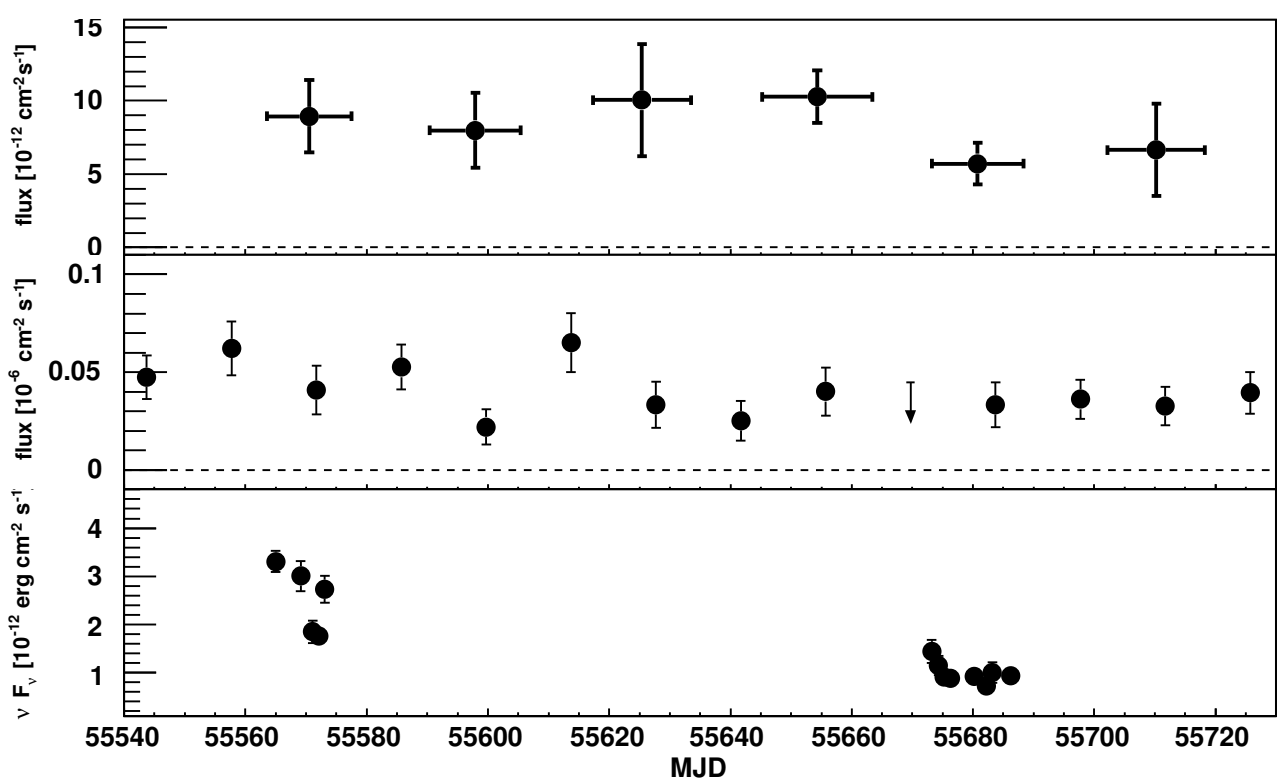

FIGURE 1. Light curves of B2 1215+30 for the first half of 2011: (top) monthly binned VERITAS light curve $(\mathrm{E}>200 \mathrm{GeV})$, (middle) bi-weekly Fermi-LAT light curve (E $>200 \mathrm{MeV})$, (bottom) Swift-XRT light curve $(2-10 \mathrm{keV})$.

rays) is interpreted as synchrotron emission from relativistic electrons in a spherical emission region, moving relativistically along the jet, which is closely aligned with the line-of-sight. The high-energy gamma-ray emission is produced via Compton upscattering off the same electron population which produced the synchrotron peak. The non-thermal electron distribution in the emission region is determined self-consistently as an equilibrium between injection of a power-law distribution with index $\alpha_{e}$, radiative cooling, and escape.

The SEDs are well described by the SSC model (see Fig. 2). For a redshift $\mathrm{z}=0.130$ the bulk Lorentz factor is $\Gamma=30$ while the emission region radius is $\sim 10^{17} \mathrm{~cm}$. The two different flux levels in X-ray are accounted for by changing the electron injection spectral index and the magnetic field strenght. The magnetic field stranght is $0.02 \mathrm{G}$ for January and $0.01 \mathrm{G}$ for April, respectively. The electron injection spectral index is $\alpha_{e}=2.8$ (Jan) and $\alpha_{e}=3.4$ (Apr) and is tightly constrained by the X-ray spectrum.

If relativistic shock acceleration takes place within the jet, the change of the electron distribution may be explained by a change in the shock field obliquity [13]. Within the modeling, the different X-ray states lead also to flux variations in the high-energy peak. Nevertheless, those could not been detected by Fermi-LAT or VERITAS given their sensitivity within the observation period reported here.

For $\mathrm{z}=0.237$ the SSC modeling is more challenging. Indeed, a higher Lorentz factor $(\Gamma=50)$ is required and the predicted VHE flux is below the VERITAS measurement. However, given the statistical and systematic errors on the energy spectrum of the highenergy peak, this redshift cannot be excluded within the framework of this SCC model. 


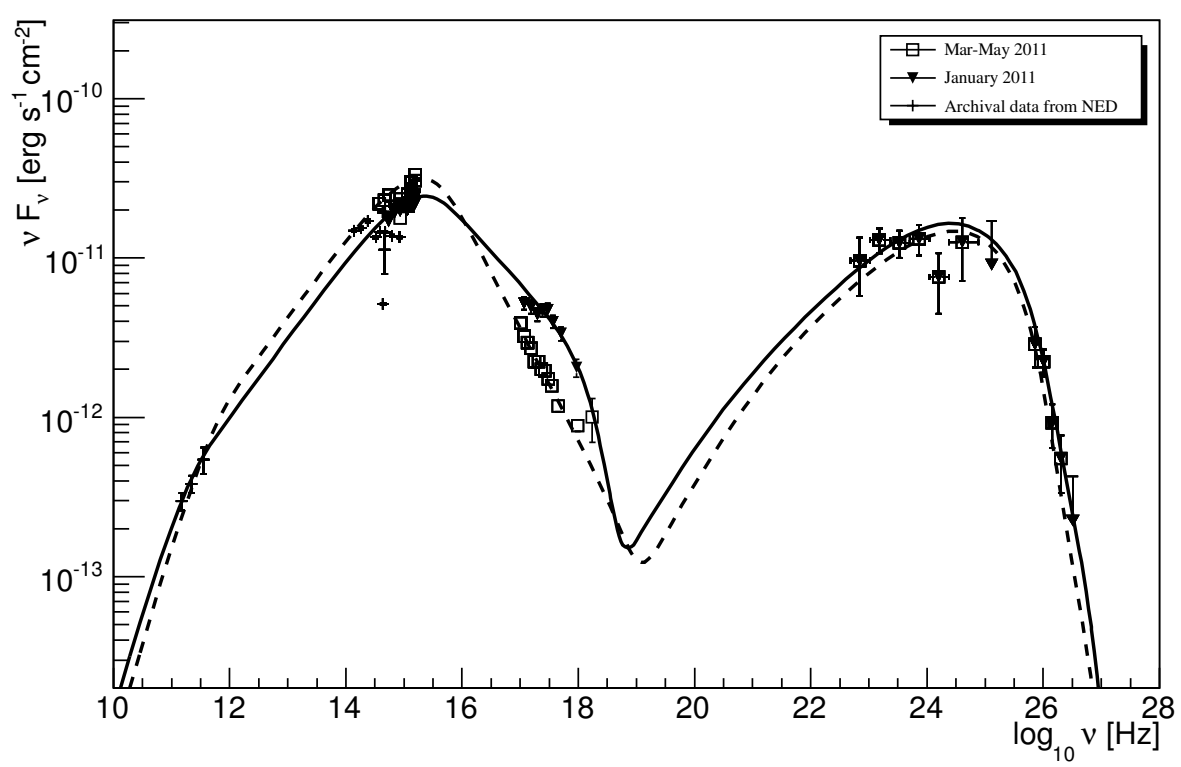

FIGURE 2. Spectral energy distribution of B2 $1215+30$. The solid line is the SSC model for the Jan 2011 data set, the dashed line represents the model for Apr 2011 incl. MDM data from May.

\section{ACKNOWLEDGMENTS}

This research is supported by grants from the U.S. Department of Energy Office of Science, the U.S. National Science Foundation and the Smithsonian Institution, by NSERC in Canada, by Science Foundation Ireland (SFI 10/RFP/AST2748) and by STFC in the U.K. We acknowledge the excellent work of the technical support staff at the Fred Lawrence Whipple Observatory and at the collaborating institutions in the construction and operation of the instrument. H. P. acknowledges support through the Young Investigators Program of the Helmholtz Association.

\section{REFERENCES}

1. Browne et al., Nature 231, 515 (1971).

2. Akiyama et al., ApJS 148, 275 (2003).

3. White et al., ApJS 126, 133 (2000).

4. Mariotti, The Astronomer's Telegram 3100 (2011).

5. Galante et al. (VERITAS Collaboration), these proceedings.

6. Li and Ma, ApJ 272, 317 (1983).

7. Aleksic et al. (MAGIC Collaboration), arxiv:1203.0490 (2012).

8. Nolan et al., ApJS 199, 31 (2012).

9. Kalberla et al., $A \& A$ 440, 775 (2005).

10. http://ned.ipac.caltech.edu/

11. Böttcher and Chiang, ApJ 581, 127 (2002).

12. Finke, Razzaque, and Dermer, ApJ 712, 238 (2010).

13. Summerlin and Baring, ApJ 745, 63 (2012). 